\title{
307 Development of mobile robot and localization system
}

\begin{abstract}
Khairul Anuar Juhari
Universiti Teknikal Malaysia Melaka Universiti Teknikal Malaysia Melaka

Teruaki Ito

The University of Tokushima

This paper describes about the development of mobile robot and localization system. The mobile robot mechanical system is build based on 3 wheels type and it includes of 5 units of ultrasonic sensors and dead reckoning sensor at each drive wheel. The mobile robot used skid steered method for its mobility. The ultrasonic sensors reading are filtered using Extended Hessian Normal Form (EHNF) to represent a model plane. Based on UMBMark method, the encoder is tune to reduce the systematic error and non-systematic error. Finally, the localization of the mobile robot is based on Extended Kalman Filter (EKF) method to estimate the mobile robot position in a global map.
\end{abstract}

\section{Key Words: Extended Hessian Normal Form (EHNF) method, UMBMark, mobile robot}

\section{Introduction}

Mobile robotics is a young field. It includes many engineering and science disciplines from mechanical, electrical and electronics engineering to computer, cognitive and social sciences [1]. For example, an autonomous mobile robot can move on its own without using any humans interfere [2]. These capabilities are called localization. There are many kinds of localization method used nowadays and one that method is the Extended Kalman Filter Localization (EKF) where the ultrasonic sensor reading and encoder reading are combined together.

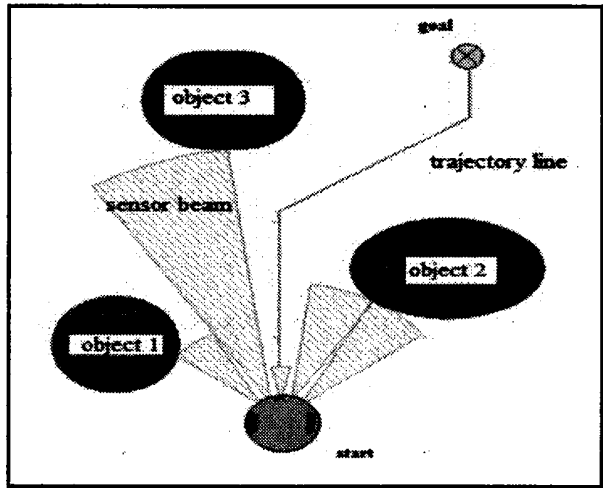

Fig.1: Experimental layout for EKF localization [1]

\section{Overview}

The main aim of this study is to develop a navigation system for unmanned ground vehicle where the robot can predict its positions in global frame using combinations of different sensors which is done using pre-processing methods. The pre-processing method will process the sensor data and reduce the error in trajectory and improve the sonar sensor readings. Then localization process will be proceeding by using the previous data which combined all he readings includes error and compile it as a value that can be used to determine the position estimation.

The kinematic model of the mobile robot used in the experiments here reported is now discussed. The mobile robot is a unicycle-like differential drive platform whose control signals are the linear and angular velocities.

\section{Extended Kalman Filter Localization}

Localization is the ability of the robot to locate it position based on global coordinate. Normally, dead reckoning sensor is used as localization tools because it can measures how far the robot moves from its stationary, but this will not precisely predict the actual pose of the robot because dead reckoning cannot measures the heading of robot in global coordinate. In order to combine the dead reckoning and ultrasonic sensors, the Extended Kalman Filter (EKF) is used. EKF methodology involved of the state prediction and the state update.

Fig.2 shows the EKF flow process for the localization system of mobile robot. In the global coordinate, the landmark has been used for localization. The EKF method requires the Kalman (k), gain that is used to predict the state of mobile robot position. Assume that the robot position is determined by Equation 1 and its best position estimate is $p^{\prime}(k \mid k)$.

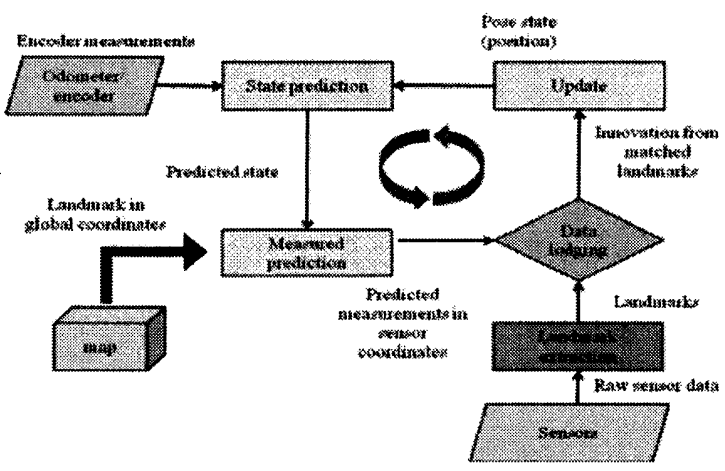

Fig.2: EKF localization process

$p(k)=[x(k) y(k) \theta(k)]^{T}$

Using Equation 1, the best estimated position of the mobile robot is given by Equation 2 .

$p^{\prime}(k+1 \mid k)=p^{\prime}(k \mid k)+u(k)=p^{\prime}(k \mid k)+\left[\begin{array}{c}\frac{\Delta s_{r}+\Delta s_{l}}{2} \cos \left(\theta+\frac{\Delta s_{r}-\Delta s_{l}}{2 b}\right) \\ \frac{\Delta s_{r}+\Delta s_{l}}{2} \sin \left(\theta+\frac{\Delta s_{r}-\Delta s_{l}}{2 b}\right) \\ \frac{\Delta s_{r}-\Delta s_{l}}{b}\end{array}\right]$

$\Sigma_{u}=\operatorname{cov}\left(\Delta s_{r}, \Delta s_{l}\right)=\left[\begin{array}{cc}k_{r}\left|\Delta s_{r}\right| & 0 \\ 0 & k_{l}\left|\Delta s_{l}\right|\end{array}\right]$ 
The covariant matrix is represented by the Equation $5 . u(k)$ is the control input that drives the robot to the position $p^{\prime}(k+1)$ The updated covariance is given by,

$\Sigma_{p}(k+1 \mid k)=\nabla_{p} f \cdot \Sigma_{p}(k \mid k) \cdot \nabla_{p} f^{T}+\nabla_{u} f \cdot \Sigma_{u}(k) \cdot \nabla_{u} f^{T}$

Where,

$R_{t}^{i}=\left[\begin{array}{ll}\sigma_{\alpha \alpha} & \sigma_{\alpha r} \\ \sigma_{r \alpha} & \sigma_{r r}\end{array}\right]_{t}^{i}$

Where:

$R_{t}^{i}=$ covariant matrix

The translation from $\{W\}$ to $\{R\}$ can be calculated as follow, $\hat{z}_{t}=\{w\}\left[\begin{array}{c}\hat{\alpha}^{j} \\ \alpha_{t} \\ \hat{r}_{t}\end{array}\right] \rightarrow \hat{r}_{t}^{j}=\{r\}\left[\begin{array}{c}\hat{\alpha}^{j} \\ \alpha_{t} \\ \hat{r}^{j} \\ r_{t}\end{array}\right]$

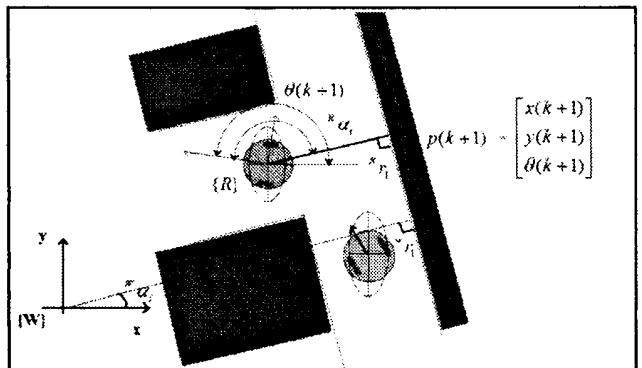

Fig.3: Localization of mobile robot in global coordinate frame

Where,

^)

$z_{t}=$ Prediction of expected features in global

$\wedge j$

$r_{t}=$ Prediction of expected features in mobile robot frame.

The translation is completed by using Equation 7,

$\hat{z}_{t}^{j}=\left[\begin{array}{c}\hat{\alpha}_{t}^{j} \\ \hat{r}_{t}^{j}\end{array}\right]=h^{j}\left(\hat{x}_{t}, m^{j}\right)=\left[\begin{array}{c}\hat{\imath}_{t}-\hat{\theta}_{t} \\ \langle w| \\ r_{t}^{j}-\left(x_{t} \cos \left({ }^{(w)} \hat{\alpha}_{t}^{j}\right)+\hat{y} \sin \left({ }^{(w)} \hat{\alpha}_{t}\right)\right.\end{array}\right]_{(7)}$

Where,

$m^{\prime}=\left\{\begin{array}{l}\{w\} \\ \alpha_{t}^{j} \\ r_{t}^{j}\end{array}\right]$

And the Jacobian matrix as follow,

$H^{j}=\left[\begin{array}{lll}\frac{\partial \alpha_{t}^{\prime}}{\partial \dot{x}} & \frac{\partial \alpha_{t}^{\prime}}{\partial \hat{y}} & \frac{\partial \alpha_{t}^{\prime}}{\partial \hat{\theta}} \\ \frac{\partial r_{t}^{\prime}}{\partial x} & \frac{\partial r_{t}^{j}}{\partial \hat{y}} & \frac{\partial r_{t}^{\prime}}{\partial \hat{\theta}}\end{array}\right]=\left[\begin{array}{cccc}0 & 0 & -1 \\ -\cos \left({ }^{\{w\}} \alpha_{t}^{j}\right) & -\sin \left({ }^{(w)} \alpha_{t}^{j}\right) & 0\end{array}\right]$

And the innovation is,

$$
\begin{aligned}
& v_{t}^{j j}=\left[z_{t}^{j}-\hat{z}_{t}^{j}\right]=\left[z_{t}^{i}-h^{j}\left(\hat{x}_{t}, m^{j}\right)\right]=\left[\begin{array}{l}
\alpha_{t}^{j} \\
r_{t}^{j}
\end{array}\right]- \\
& {\left[\begin{array}{c}
{ }^{\{\omega\}} \alpha_{t}^{j}-\hat{\theta}_{t} \\
{ }^{\{\omega\}} r_{t}^{j}-\left(\hat{x}_{t} \cos \left({ }^{\{\omega\}} \alpha_{t}^{j}\right)+\hat{y}_{t} \sin \left({ }^{\{\omega\}} \alpha_{t}^{j}\right)\right)
\end{array}\right.}
\end{aligned}
$$

Finally the estimated position based on EKF is,

$\Sigma_{I N t}^{j}=H^{j} \cdot \hat{P}_{t} \cdot H^{j T}+R_{t}^{i}$

Where the predicted new position is,

$p^{\prime}(k \mid k)=\left[\begin{array}{c}x_{i}^{j} k \\ y_{i}^{j} k \\ \theta_{i}^{j} k\end{array}\right]$

As shown in Fig.4, the red circle shows the predicted position of the mobile robot after using EKF.

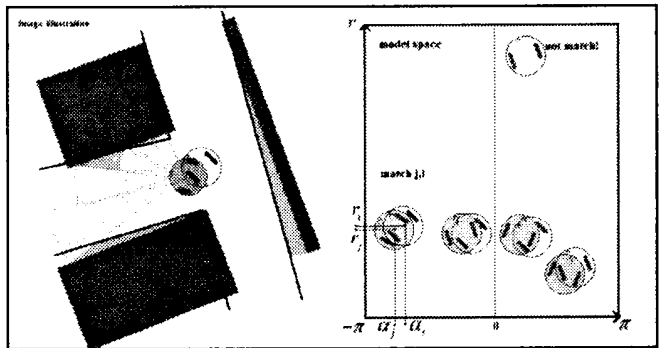

Fig.4: Predicted position mobile robot based on EKF method

\section{Summary}

Localization of mobile robot requires the mobile robot to locate itself in the global map where the artificial landmark has been used. The EHNF method has been used to identify the geometrical shape of the unstructured environment known as target model. These target models are divided into different classes such as plane, cylinder, corner and edge. In order to locate the position, the mobile robot must be able to localize its position using a method called EKF localization. At the end, the estimated position of the UGV is based on EKF where the $p^{\prime}(k \mid k)$ is observed.

\section{References}

[1] Zang, Y.Z., 2012. Localization and Tracking of Indoor Mobile Robot with Ultrasonic and Dead-reckoning Sensors, Journal of Computational Information Systems 8(2), pp. 531-539.

[2] Bento et al., "Sensor Fusion for Precise Autonomous Vehicle Navigation in Outdoor Semi-structured Environments", IEEE Int. Conf. on Intelligent Transportation Systems (ITSC2005), Basel, Switzerland, 2005, pp.245-250.

[3] ChangBae Jung "Design of test tracks for odometry calibration of wheeled mobile robots". International Journal of advance robotic systems". Vol 8. No 4. pp 1-9. May. 2011

[4] James L. Crowley. "Asynchronous control of rotation and translation for robot vehicle". Journal of Robotics and Autonomous Systems, pp. 1-16, Aug. 1992

[5] Bostani et al., A Novel Method to Measure and Correct the Odometry Errors in Mobile Robots, IEEE Canadian Conference on Electrical and Computer Engineering, Niagara Falls, Canada, 2008. 